Published in final edited form as:

Mol Pharm. 2016 March 7; 13(3): 990-995. doi:10.1021/acs.molpharmaceut.5b00843.

\title{
Influence of Coformer Stoichiometric Ratio on Pharmaceutical Cocrystal Dissolution: Three Cocrystals of Carbamazepine/4- Aminobenzoic Acid
}

\author{
Zi Li and Adam J. Matzger ${ }^{*}$ \\ Department of Chemistry, Macromolecular Science and Engineering Program, University of \\ Michigan, 930 North University Avenue, Ann Arbor, Michigan 48109-1055, United States
}

\begin{abstract}
Cocrystallization is a technique to optimize solid forms that shows great potential to improve the solubility of active pharmaceutical ingredients (APIs). In some systems, an API can form cocrystals in multiple stoichiometries with the same coformer. However, it remains unclear how coformer stoichiometry influences solubility. This paper investigates the pharmaceutical:coformer pair carbamazepine (CBZ)/p-aminobenzoic acid (PABA); both CBZ/PABA 1:1 and 2:1 cocrystals are known, and a novel 4:1 CBZ/PABA cocrystal is reported here. The 4:1 cocrystal is structurally characterized, and phase stability data suggest that it is a thermodynamically unstable form.

Dissolution experiments show that there is no correlation between the cocrystal stoichiometry and dissolution rate in this system. On the other hand, with the relatively weak intermolecular interactions, metastable forms can be beneficial to dissolution rate, which suggests that more effort should be devoted to cocrystal production with kinetic growth methods.
\end{abstract}

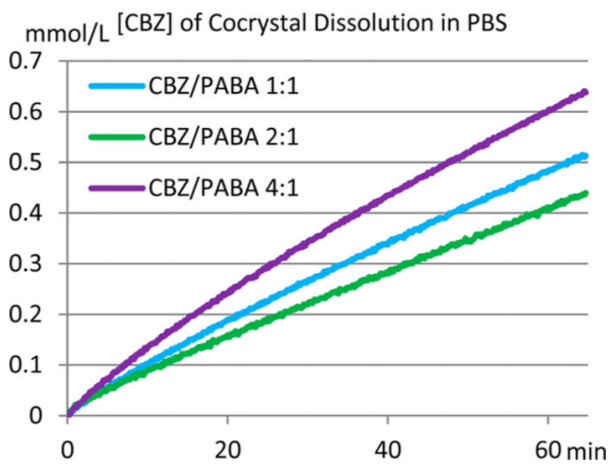

\section{Keywords}

cocrystallization; coformer stoichiometry; stability; phase conversion; solubility; dissolution

\footnotetext{
*Corresponding Author matzger@umich.edu.

The authors declare no competing financial interest.

ASSOCIATED CONTENT

Supporting Information

The Supporting Information is available free of charge on the ACS Publications website at DOI: 10.1021/acs.molpharmaceut.5b00843.

Experimental details (PDF)

Crystallographic data (CIF)
} 


\section{INTRODUCTION}

Active pharmaceutical ingredients (APIs) that suffer from low aqueous solubility (BCS class II and IV) display slow dissolution rates, and in many cases this slow drug release from dosage forms hinders the bioavailability of the compounds. ${ }^{1,2}$ Multiple approaches aimed at optimizing the solid form to overcome solubility-limited bioavailability have been investigated due to the increasing proportion of poorly soluble drugs being developed. For crystalline drugs, the neutral API form, salts, and multicomponent crystals, such as solvates and cocrystals, can be considered for development into a dosage. ${ }^{3-8}$ Among these approaches, cocrystallization is an attractive option for solid form optimization of nonionizable drugs for which salt formation is not applicable. ${ }^{9-11}$ Cocrystallization can dramatically alter the properties of neutral compounds by introducing a neutral coformer that interacts with the target API in a defined stoichiometric ratio through intermolecular interactions; this approach presents great potential to adjust API solubility. Similar to salt formation, cocrystallization can increase both solubility and dissolution rate of drugs. ${ }^{8}$ The overall effect of a coformer on cocrystal solubility depends on both the solvation energies of the cocrystallizing components and the cocrystal lattice energy. ${ }^{12}$ As a result, a soluble coformer can promote cocrystal dissolution with strong solvent-solute interactions formed from solvation diving supersaturation of the API; in this way cocrystals can improve the bioavailability of poorly soluble drugs. ${ }^{13}$

In some cocrystallization systems, multiple cocrystal stoichiometries are possible with the same coformer and API, which, in principle, provides more opportunities to engineer dissolution behavior. Studies on the synthons in such systems as well as the conversion between different cocrystal stoichiometries have been reported. ${ }^{14-21}$ It was revealed that cocrystals of different stoichiometric ratios can coexist in solution at a eutectic point, and the conversion between cocrystal stoichiometries in solution is dependent on the coformer concentration. What is not understood is how cocrystal stoichiometry influences API performance.

For cocrystals where multiple stoichiometries are available with the same coformer, as the stoichiometry of a more soluble coformer increases, the enhanced capacity to impart high solubility is expected to favor cocrystal dissolution. ${ }^{22}$ On the other hand, as an analogue of the common ion effect in salts, the drug concentration of cocrystals at equilibrium decreases as a function of increasing cocrystallizing component concentration in solution. ${ }^{15,23}$ Consequently, for cocrystals with greater coformer stoichiometry, the coformer concentration in solution is higher for a given drug concentration, which may favor drug precipitation in alternative stoichiometries. Another property of cocrystals with higher coformer stoichiometry is the greater mass of material required to reach the same API concentration. The above analysis indicates that there are multiple factors imposed by coformer stoichiometry that influence cocrystal dissolution and the relative influence of each of these is not well-defined. These are, however, not the only factors of significance as intermolecular interactions among the cocrystals may vary in both strength and abundance (vide infra). Multiple conformer stoichiometries have previously been observed to lead to changes in drug solubilization albeit in a system where the changes in conformer 
stoichiometry are accompanied by changes in cocrystal solvation (both solvent identity and stoichiometry) thus convoluting the conformer stoichiometry effects with solvation effects. ${ }^{24}$

Carbamazepine (CBZ, Figure 1) is a polymorphic anti-epileptic BCS class II compound and therefore exhibits low aqueous solubility and high permeability. ${ }^{1,25}$ Efforts have been made to optimize CBZ solid form solubility and dissolution rate through cocrystallization. ${ }^{26-28} p$ Aminobenzoic acid (PABA, Figure 1) is a coformer exhibiting an aqueous solubility of $\sim 5.0$ $\mathrm{g} / \mathrm{L}$ at $24{ }^{\circ} \mathrm{C}$, a value much higher than that for CBZ $(17.7 \mathrm{mg} / \mathrm{L}) .{ }^{29,30}$ A CBZ/PABA 1:1 cocrystal and a CBZ/PABA 2:1 cocrystal have been described previously. ${ }^{15,31,32}$ Here, a new stoichiometry CBZ/PABA 4:1 cocrystal is reported. With more than two different cocrystal stoichiometries structurally characterized, a result rarely found in cocrystallized pharmaceuticals, ${ }^{20,21} \mathrm{CBZ} / \mathrm{PABA}$ is an attractive system to study the influence of multiple stoichiometric ratios on materials properties and, in particular, how the stoichiometric ratio of a soluble coformer affects the dissolution of a drug.

\section{RESULTS AND DISCUSSION}

\subsection{CBZ/PABA 4:1 Cocrystal Characterization}

Clusters of needles were obtained by solvent evaporation of CBZ/PABA ethanolic solutions of various stoichiometric ratios. Due to the similarities in morphology of the known CBZ I, CBZ II, PABA I, and CBZ/PABA 1:1 cocrystal, there is no reliable way to visually distinguish among the needle-shaped crystals; therefore powder X-ray diffraction (PXRD) characterization was performed for form identification of obtained needles. From samples crystallized out of a $0.2 \mathrm{M} \mathrm{CBZ:0.1} \mathrm{M} \mathrm{PABA} \mathrm{ethanol} \mathrm{solution,} \mathrm{as} \mathrm{well} \mathrm{as} \mathrm{a} \mathrm{0.1} \mathrm{M} \mathrm{CBZ:0.1}$ M PABA ethanol solution, a unique PXRD pattern was observed, with characteristic lines at $2 \theta=6.63,8.71$, and $13.30^{\circ}$, indicating a novel crystalline form. Raman spectroscopy characterization was conducted to determine the constituents of the new form. Similarly to the known CBZ/PABA cocrystals, the Raman spectrum of the new form exhibits peaks in regions where $\mathrm{CBZ}$ or PABA single component crystals show characteristic peaks, but these peak positions are distinguishable from the spectra of all the previously reported forms (Figure 2). These features led to the speculation that the new form is a novel CBZ/PABA cocrystal.

To determine the stoichiometry of the newly discovered cocrystal of CBZ/PABA, proton NMR spectroscopy was carried out and a 4:1 CBZ:PABA ratio was measured. This new phase is CBZ-rich relative to the previously reported CBZ/PABA 1:1 and 2:1 forms, and this result agrees with the Raman spectrum of the new form in which the features of PABA between 800 and $900 \mathrm{~cm}^{-1}$ are relatively weak compared to those of CBZ/PABA 1:1 and 2:1 cocrystals. Single crystal XRD reveals that the CBZ/PABA 4:1 cocrystal is monoclinic in the space group $\mathrm{C} 2 / c$ (Table 1). Although severe PABA disorder makes detailed analysis of intermolecular interactions between the components in the 4:1 cocrystal impossible, packing of the three cocrystals is sufficiently different that motifs responsible for each stoichiometry are easily discerned. The 4:1 cocrystal packing features infinite PABA chains occupying channels between isolated homodimers of CBZ; all CBZ molecules are symmetry equivalent, and this is distinct from the previously reported forms where interactions between CBZ and PABA are present. The 1:1 cocrystal is characterized by infinite chains of 
CBZ homodimers connected to PABA homodimers by interaction by $\mathrm{N}-\mathrm{H}$ donation from PABA to CBZ carbonyls. All CBZ molecules are symmetry equivalent in the 1:1 cocrystal. The 2:1 cocrystal is characterized by finite groups of two heterodimerized CBZ-PABA pairs connected to one CBZ homodimer by $\mathrm{N}-\mathrm{H}$ (PABA)/carbonyl (CBZ) interactions. This leads to two symmetry inequivalent CBZ molecules (Figure 3).

\subsection{Stability Evaluation}

The CBZ/PABA 4:1 cocrystal shows behavior consistent with a metastable form. CBZ/ PABA 1:1 and 2:1 cocrystals can be prepared on large scale by neat grinding, solventassisted grinding with ethanol, or slurring CBZ with corresponding stoichiometric ratio equivalents of PABA. These results are consistent with the notion that CBZ/PABA 1:1 and 2:1 cocrystals are thermodynamically stable forms of the corresponding CBZ/PABA stoichiometries. On the other hand, by neat grinding, solvent-assisted grinding, and slurring of CBZ with 0.25 molar equiv of PABA, the desired CBZ/PABA 4:1 cannot be obtained, and instead physical mixtures of CBZ III and CBZ/PABA 2:1 form. This holds true either in the absence or in the presence of seeds of the 4:1 cocrystal, indicating that the result is not kinetic. Additionally, wetted by a minimum amount of either pure solvent or filtered solution cosaturated with CBZ and CBZ/PABA 2:1 cocrystal, 4:1 cocrystals also convert to physical mixtures of CBZ III and CBZ/PABA 2:1. The spontaneous conversion of 4:1 samples to crystals of alternative stoichiometry suggests that, unlike the 1:1 and 2:1 cocrystals, the 4:1 cocrystal is not a thermodynamically favored form relative to the physical mixture of CBZ/ PABA 2:1 and CBZ III. Despite the fact that CBZ/PABA 4:1 cocrystals cannot be obtained from grinding or slurring, once the cocrystals are formed, they can be stored without decom position or conversion to other forms under ambient temperature and humidity, and only convert once solvent is present.

In the reported phase diagram for the CBZ/PABA/ethanol system, the CBZ/PABA 1:1 cocrystal has invariant points with either CBZ/PABA 2:1 or PABA, and the CBZ/PABA 2:1 cocrystal has invariant points with either CBZ or CBZ/PABA 1:1. ${ }^{15}$ If the $4: 1$ cocrystal is present on this phase diagram, it would be in a region somewhere between pure $\mathrm{CBZ}$ and pure CBZ/PABA 2:1. However, all CBZ/PABA 4:1 cocrystals suspended in slurries of pure CBZ, pure CBZ/PABA 2:1, or mixtures of CBZ and CBZ/PABA 2:1 dissolved in agreement with experiments of CBZ/PABA 4:1 conversion to CBZ and CBZ/PABA 2:1 described above; thus CBZ/PABA 4:1 does not have a stable region on the ternary phase diagram. A similar situation has been reported with a caffeine/maleic acid 2:1 cocrystal obtained by neat and solvent-assisted grinding but unattainable by crystallization from solvent; the form is metastable and converts to a mixture of caffeine and 1:1 cocrystal with the addition of acetone. ${ }^{17}$ A ciprofloxacin succinate $(2: 1)$ tetrahydrate has also been implicated to be metastable. ${ }^{17,33} \mathrm{~A}$ final example is a diacetone diperoxide/1,3,5-tribromo-2,4,6trinitrobenzene cocrystal that decomposes to the pure forms of its constituents at room temperature. ${ }^{34}$ The term "schizophilic" has been applied to describe such a cocrystal that is unstable relative to its pure components. Hence, the new CBZ/PABA 4:1 cocrystal is a schizophilic cocrystal that, instead of being unstable relative to its pure components, yields a mixture of one pure component (CBZ) and a second stoichiometry of cocrystal (CBZ/PABA $2: 1)$. 
Thermal stabilities of the three CBZ/PABA cocrystal stoichiometries were examined by differential scanning calorimetry (DSC). Both CBZ/PABA 1:1 and 2:1 cocrystals presented only one major endothermic peak, at 148 and $157^{\circ} \mathrm{C}$ respectively, corresponding to their melting points. By contrast, the 4:1 cocrystal DSC profiles revealed a phase transition at $100^{\circ} \mathrm{C}$, followed by multiple endothermic peaks (Figure 4). The transformation products at $100{ }^{\circ} \mathrm{C}$ were identified by applying hot stage Raman microspectroscopy. Changes in cocrystal morphology from prisms with smooth surfaces to rough crystals with small needles attaching to the surfaces were observed. Raman spectroscopy showed that the 4:1 cocrystal gradually disappeared at the conversion temperature and peaks of CBZ I, the preferred CBZ polymorph at high temperature, appeared. PXRD characterization of the product showed that both CBZ I and the CBZ/PABA 1:1 cocrystal formed. The DSC results indicate that CBZ/PABA 4:1 has weaker thermal stability than CBZ/PABA 1:1 and 2:1 cocrystals, and undergoes phase conversion before reaching its melting point, which is suggestive of relatively weak intermolecular interactions in the 4:1 cocrystal compared to the previously known forms.

\subsection{Dissolution Experiments}

Ultimately the goal of cocrystallization for most APIs is to improve solubility, and the question being addressed here is how stoichiometry influences this property. Because CBZ/ PABA 4:1 is metastable and converts easily to other forms in solution, dissolution rate rather than the drug concentration in the saturated solution was applied to assess the influence of coformer on solubility. Here the dissolution investigation started with the hypothesis that the drug dissolution can benefit from the increasing stoichiometry of a more soluble coformer, and that the CBZ/PABA cocrystallization system, with three different cocrystal stoichiometries accessible, serves as a model system for the investigation of this proposal.

$\mathrm{CBZ}$ and PABA solutes enter the solution during cocrystal dissolution. Therefore, the dissolution behaviors of all cocrystals are described by the changes in concentration of both components in the dissolution media, which are calculated based on the UV-vis spectra applied to monitor the dissolution process. Detailed derivation of equations used to treat data and dissolution profiles are included in the Supporting Information.

2.3.1. Dissolution Experiments in Phosphate-Buffered Saline (PBS)—With PBS as the aqueous dissolution medium, it was noted that, in all experiments of samples containing CBZ, including CBZ III, CBZ dihydrate, and all CBZ/PABA cocrystals, the dissolution rates, represented by the slope of the concentration-time profiles, kept decreasing over time during the dissolution process. Additionally, congruent dissolution of CBZ/PABA cocrystals was never observed during the dissolution process, and cocrystal dissolution behavior can only be described by the concentration of both components separately. Therefore, the dissolution behavior of CBZ III, CBZ hydrate, CBZ/PABA 1:1, CBZ/PABA 2:1, and CBZ/PABA 4:1 in PBS is described by the initial CBZ and PABA dissolution rates and the dissolution rates after $1 \mathrm{~h}$ (Table 2).

In opposition to the notion that an increase of the PABA stoichiometry would promote drug dissolution, CBZ/PABA 4:1, the cocrystal with the lowest PABA stoichiometric ratio, 
exhibited the highest CBZ dissolution rate at all times, whereas CBZ/PABA 2:1 showed the lowest CBZ dissolution rate among the three cocrystals. Notably, for all CBZ/PABA cocrystal samples, the ratios of CBZ dissolution rates compared to PABA dissolution rates are always lower than the CBZ:PABA cocrystal stoichiometry. This result indicates that CBZ/PABA cocrystal dissolution in PBS is not a simple one-step process; either the two cocrystal components do not dissolve congruently or they form conversion, and/or precipitation occurs during dissolution.

In order to elucidate possible solid form conversion during dissolution, in situ Raman spectroscopy and ex situ PXRD characterization were applied to examine solid form present in the dissolution pellet. Formation of CBZ dihydrate on the surface of CBZ III and all CBZ/ PABA cocrystals was observed by in situ Raman spectroscopy during dissolution. These observations can be rationalized by generation of CBZ supersaturation with respect to CBZ dihydrate in the dissolution layer leading to precipitation on the sample surface; such observations mirror those reported in the cases of CBZ/nicotinamide and CBZ/saccharin cocrystals. ${ }^{27,35}$ This outcome is supported by analysis of the remaining samples after $1 \mathrm{~h}$ of dissolution in which physical mixtures of the original form and CBZ dihydrate were identified by both Raman spectroscopy and PXRD. With CBZ dihydrate covering the sample surface, a smaller effective dissolution surface area of the original form is obtained, resulting in a drop in CBZ III and CBZ/PABA cocrystal dissolution rates. For the same reasons, the $\mathrm{CBZ}$ and PABA dissolution rates measured do not track the cocrystal stoichiometry, since part of the dissolved CBZ in the dissolution layer precipitates on the pellet surface as CBZ dihydrate. This is not the sole effect reducing dissolution rate with time as the concentration of CBZ in the dissolution medium is sufficient to be influencing the dissolution rates (this is evident for CBZ dihydrate). These effects are smaller during initial dissolution, and so the behavior of the CBZ component more closely mirrors the intrinsic properties of samples. In these cases CBZ dissolution rates increase in the order CBZ/PABA 2:1 < CBZ/PABA 1:1< CBZ/PABA 4:1. Clearly there is no correlation between the cocrystal stoichiometry and dissolution rate.

2.3.2. Dissolution Experiments in Acetonitrile-In order to obtain dissolution profiles directly mirroring sample properties without the disturbance of phase conversion to CBZ dihydrate, dissolution experiments of all samples were conducted in acetonitrile. In acetonitrile at $20^{\circ} \mathrm{C}$ the solubility of CBZ III is $35.2 \mathrm{~g} / \mathrm{L}$ and that of PABA I is $62.2 \mathrm{~g} / \mathrm{L} .{ }^{36,37}$ PABA still exhibits higher solubility than CBZ, but compared to the case in PBS the difference in solubility is much smaller. In contrast to observations in PBS, the CBZ and PABA dissolution profiles in acetonitrile were linear with constant slope (within the limit of instrument linearity) corresponding to the dissolution rate indicating constant dissolution behavior. In addition, CBZ and PABA dissolution rates of all cocrystal samples in acetonitrile mirror the cocrystal stoichiometric ratios (Table 3), indicating that both components dissolved congruently. The hypothesis that CBZ dissolution can be improved by the increase of PABA stoichiometry was again disproved, since CBZ/PABA 4:1, the cocrystal with the lowest PABA stoichiometric ratio, again presented the highest drug dissolution rate. The dissolution rate of $\mathrm{CBZ}$ in CBZ/PABA 2:1, which might be expected to 
be higher than that of pure CBZ III because of the presence of PABA coformer, turns out to be the lowest among all samples.

With regard to the dissolution behavior of CBZ/PABA cocrystals in acetonitrile, CBZ/PABA 4:1 showed the highest CBZ dissolution rate, which is attributed to the relatively weak intermolecular interactions implied by its schizophilic behav iors. CBZ/PABA 2:1 presented the lowest dissolution rate in both PBS and acetonitrile dissolution experiments. In fact CBZ/PABA 2:1 does not display improved drug dissolution in acetonitrile relative to CBZ III; this result can be attributed to the fact that PABA solubility in acetonitrile is not significantly higher than that of CBZ (minimal differential driving force for dissolution) and thus the dissolution disadvantage resulting from the strong intermolecular interactions in the 2:1 cocrystal could not be overcome by the small advantage imparted by improved PABA solubility.

\section{CONCLUSIONS}

A CBZ/PABA cocrystal of 4:1 stoichiometry has been discovered and structurally characterized. Stability studies showed that the novel form is schizophilic and converts readily to a mixture of pure $\mathrm{CBZ}$ and alternative stoichiometries of CBZ/PABA upon being wetted by solvent. Although cocrystallization is known to be an applicable technique to improve the solubility and dissolution properties of poorly soluble drugs, in the dissolution experiments of CBZ/PABA cocrystals in PBS and acetonitrile, it is demonstrated here that complex, and cocrystallization with a more soluble coformer does not necessarily improve drug dissolution. In particular when the difference in solubility between the drug and the coformer is not large (in acetonitrile), the intermolecular interactions within the crystal lattice are a critical factor influencing cocrystal dissolution. Therefore, the stoichiometry of a soluble coformer cannot be used as a straightforward criterion to estimate the cocrystal dissolution behavior, and consequently, increasing coformer stoichiometry in a cocrystal is not a reliable approach to optimizing drug solubility and dissolution. On the other hand, greater attention to kinetic growth methods should be paid for cocrystal screening in attempting to optimize bioavailability of poorly soluble drugs, since crystal dissolution can benefit from the weak intermolecular interactions in the thermodynamically unstable forms.

\section{Supplementary Material}

Refer to Web version on PubMed Central for supplementary material.

\section{ACKNOWLEDGMENTS}

This work was financially supported by the National Institutes of Health Grant No. RO1 GM106180. Thanks are extended to Dr. Jeff W. Kampf for single crystal analysis and Dr. Naír Rodríguez-Hornedo for discussion on cocrystal stability and dissolution experiments.

\section{REFERENCES}

1. Zakeri-Milani P, Barzegar-Jalali M, Azimi M, Valizadeh H. Biopharmaceutical classification of drugs using intrinsic dissolution rate (IDR) and rat intestinal permeability. Eur. J. Pharm. Biopharm. 2009; 73:102-106. [PubMed: 19442726] 
2. Amidon G, Lennernäs H, Shah V, Crison J. A Theoretical Basis for a Biopharmaceutic Drug Classification: The Correlation of in Vitro Drug Product Dissolution and in Vivo Bioavailability. Pharm. Res. 1995; 12:413-420. [PubMed: 7617530]

3. Berge SM, Bighley LD, Monkhouse DC. Pharmaceutical salts. J. Pharm. Sci. 1977; 66:1-19. [PubMed: 833720]

4. Rodríguez-Spong B, Price CP, Jayasankar A, Matzger AJ, Rodríguez-Hornedo N. General principles of pharmaceutical solid polymorphism: A supramolecular perspective. Adv. Drug Delivery Rev. 2004; 56:241-274.

5. Serajuddin ATM. Salt formation to improve drug solubility. Adv. Drug Delivery Rev. 2007; 59:603616.

6. Babu NJ, Nangia A. Solubility Advantage of Amorphous Drugs and Pharmaceutical Cocrystals. Cryst. Growth Des. 2011; 11:2662-2679.

7. Kawabata Y, Wada K, Nakatani M, Yamada S, Onoue S. Formulation design for poorly watersoluble drugs based on biopharmaceutics classification system: Basic approaches and practical applications. Int. J. Pharm. 2011; 420:1-10. [PubMed: 21884771]

8. Elder DP, Holm R, Diego H. L. d. Use of pharmaceutical salts and cocrystals to address the issue of poor solubility. Int. J. Pharm. 2013; 453:88-100. [PubMed: 23182973]

9. Almarsson O, Zaworotko MJ. Crystal engineering of the composition of pharmaceutical phases. Do pharmaceutical co-crystals represent a new path to improved medicines? Chem. Commun. 2004:1889-1896.

10. Qiao N, Li M, Schlindwein W, Malek N, Davies A, Trappitt G. Pharmaceutical cocrystals: An overview. Int. J. Pharm. 2011; 419:1-11. [PubMed: 21827842]

11. Thakuria R, Delori A, Jones W, Lipert MP, Roy L, Rodríguez-Hornedo N. Pharmaceutical cocrystals and poorly soluble drugs. Int. J. Pharm. 2013; 453:101-125. [PubMed: 23207015]

12. Roy, L.; Lipert, MP.; Rodriguez-Hornedo, N. Co-crystal Solubility and Thermodynamic Stability.. In: Wouters, J.; Quéré, L., editors. Pharmaceutical Salts and Co-crystals. The Royal Society of Chemistry; 2012. p. 247-279.

13. Good DJ, Rodríguez-Hornedo N. Cocrystal Eutectic Constants and Prediction of Solubility Behavior. Cryst. Growth Des. 2010; 10:1028-1032.

14. Trask AV, van de Streek J, Motherwell WDS, Jones W. Achieving Polymorphic and Stoichiometric Diversity in Cocrystal Formation: Importance of Solid-State Grinding, Powder X-ray Structure Determination, and Seeding. Cryst. Growth Des. 2005; 5:2233-2241.

15. Jayasankar A, Reddy LS, Bethune SJ, Rodríguez-Hornedo N. Role of Cocrystal and Solution Chemistry on the Formation and Stability of Cocrystals with Different Stoichiometry. Cryst. Growth Des. 2009; 9:889-897.

16. Alhalaweh A, George S, Boström D, Velaga SP. 1:1 and 2:1 Urea-Succinic Acid Cocrystals: Structural Diversity, Solution Chemistry, and Thermodynamic Stability. Cryst. Growth Des. 2010; 10:4847-4855.

17. Guo K, Sadiq G, Seaton C, Davey R, Yin Q. Co-Crystallization in the Caffeine/Maleic Acid System: Lessons from Phase Equilibria. Cryst. Growth Des. 2010; 10:268-273.

18. Bevill MJ, Vlahova PI, Smit JP. Polymorphic Cocrystals of Nutraceutical Compound p-Coumaric Acid with Nicotinamide: Characterization, Relative Solid-State Stability, and Conversion to Alternate Stoichiometries. Cryst. Growth Des. 2014; 14:1438-1448.

19. He H, Jiang L, Zhang Q, Huang Y, Wang J-R, Mei X. Polymorphism observed in dapsone-flavone cocrystals that present pronounced differences in solubility and stability. CrystEngComm. 2015; 17:6566-6574.

20. Sarma B, Reddy LS, Nangia A. The Role of $\pi$-Stacking in the Composition of Phloroglucinol and Phenazine Cocrystals. Cryst. Growth Des. 2008; 8:4546-4552.

21. Zhang S-W, Harasimowicz MT, de Villiers MM, Yu L. Cocrystals of Nicotinamide and (R)Mandelic Acid in Many Ratios with Anomalous Formation Properties. J. Am. Chem. Soc. 2013; 135:18981-18989. [PubMed: 24215608]

22. Good DJ, Rodríguez-Hornedo N. Solubility Advantage of Pharmaceutical Cocrystals. Cryst. Growth Des. 2009; 9:2252-2264. 
23. Bethune SJ, Huang N, Jayasankar A, Rodríguez-Hornedo N. Understanding and Predicting the Effect of Cocrystal Components and pH on Cocrystal Solubility. Cryst. Growth Des. 2009; 9:3976-3988.

24. Saikia B, Bora P, Khatioda R, Sarma B. Hydrogen Bond Synthons in the Interplay of Solubility and Membrane Permeability/Diffusion in Variable Stoichiometry Drug Cocrystals. Cryst. Growth Des. 2015; 15:5593-5603.

25. Grzesiak AL, Lang M, Kim K, Matzger AJ. Comparison of the four anhydrous polymorphs of carbamazepine and the crystal structure of form I. J. Pharm. Sci. 2003; 92:2260-2271. [PubMed: 14603511]

26. Fleischman SG, Kuduva SS, McMahon JA, Moulton B, Bailey Walsh RD, Rodríguez-Hornedo N, Zaworotko MJ. Crystal Engineering of the Composition of Pharmaceutical Phases: MultipleComponent Crystalline Solids Involving Carbamazepine. Cryst. Growth Des. 2003; 3:909-919.

27. Hickey MB, Peterson ML, Scoppettuolo LA, Morrisette SL, Vetter A, Guzmán H, Remenar JF, Zhang Z, Tawa MD, Haley S, Zaworotko MJ, Almarsson Ö. Performance comparison of a cocrystal of carbamazepine with marketed product. Eur. J. Pharm. Biopharm. 2007; 67:112-119. [PubMed: 17292592]

28. Childs SL, Rodriguez-Hornedo N, Reddy LS, Jayasankar A, Maheshwari C, McCausland L, Shipplett R, Stahly BC. Screening strategies based on solubility and solution composition generate pharmaceutically acceptable cocrystals of carbamazepine. CrystEngComm. 2008; 10:856-864.

29. Kumar S, Bhargava D, Thakkar A, Arora S. Drug carrier systems for solubility enhancement of BCS class II drugs: a critical review. Crit. Rev. Ther. Drug Carrier Syst. 2013; 30:217-256. [PubMed: 23614647]

30. Gracin S, Rasmuson ÅC. Polymorphism and Crystallization of p-Aminobenzoic Acid. Cryst. Growth Des. 2004; 4:1013-1023.

31. McMahon JA, Bis JA, Vishweshwar P, Shattock TR, McLaughlin OL, Zaworotko MJ. Crystal engineering of the composition of pharmaceutical phases. 3. Primary amide supra-molecular heterosynthons and their role in the design of pharmaceutical co-crystals. Z. Kristallogr. - Cryst. Mater. 2005; 220:340-350.

32. A CBZ/PABA/water ternary crystal is also known but does not show up in the studies reported here. See ref 30.

33. Paluch KJ, McCabe T, Müller-Bunz H, Corrigan OI, Healy AM, Tajber L. Formation and Physicochemical Properties of Crystalline and Amorphous Salts with Different Stoichiometries Formed between Ciprofloxacin and Succinic Acid. Mol. Pharmaceutics. 2013; 10:3640-3654.

34. Landenberger KB, Bolton O, Matzger AJ. Two Isostructural Explosive Cocrystals with Significantly Different Thermodynamic Stabilities. Angew. Chem., Int. Ed. 2013; 52:6468-6471.

35. Qiao N, Wang K, Schlindwein W, Davies A, Li M. In situ monitoring of carbamazepinenicotinamide cocrystal intrinsic dissolution behaviour. Eur. J. Pharm. Biopharm. 2013; 83:415426. [PubMed: 23159709]

36. Svard M, Nordstrom FL, Hoffmann E-M, Aziz B, Rasmuson AC. Thermodynamics and nucleation of the enantiotropic compound p-aminobenzoic acid. CrystEngComm. 2013; 15:5020-5031.

37. Ikni A, Clair B, Scouflaire P, Veesler S, Gillet J-M, El Hassan N, Dumas F, Spasojević-de Biré A. Experimental Demonstration of the Carbamazepine Crystallization from Non-photochemical Laser-Induced Nucleation in Acetonitrile and Methanol. Cryst. Growth Des. 2014; 14:3286-3299. 


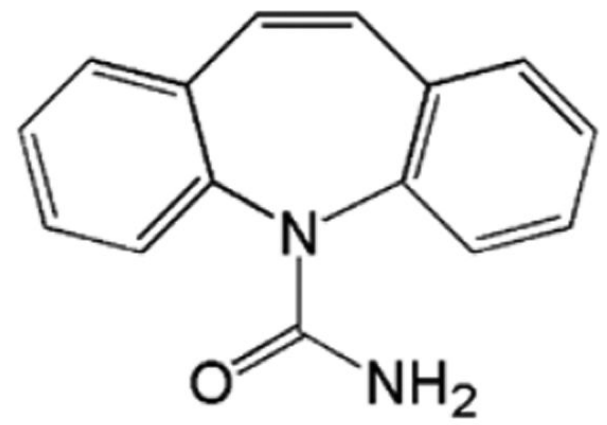

CBZ

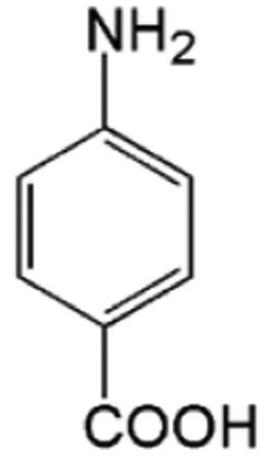

PABA

Figure 1.

Structural formulas of CBZ and PABA. 


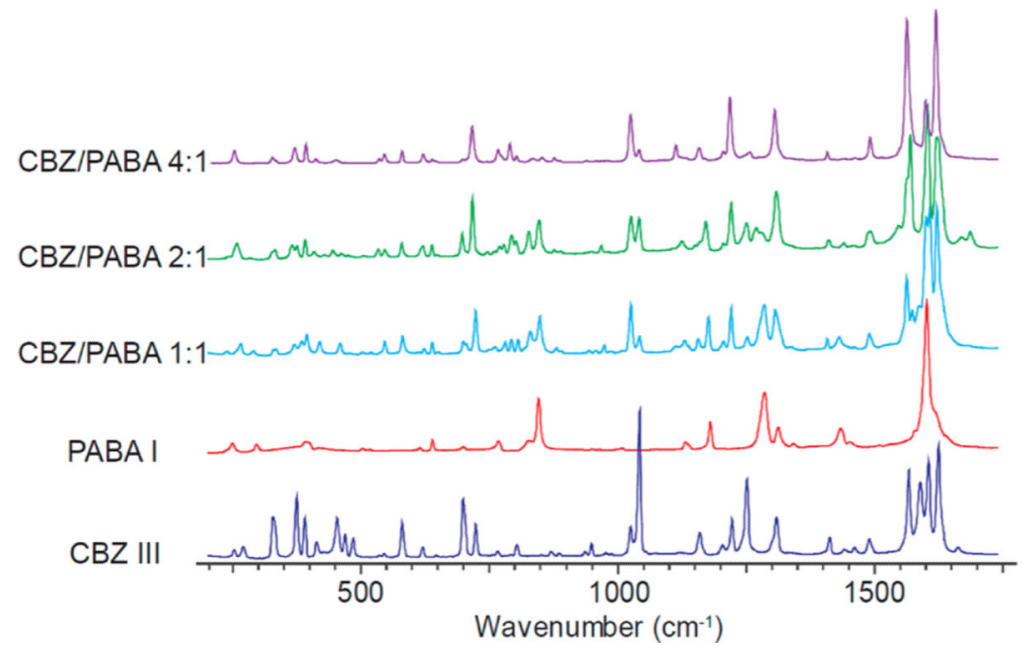

Figure 2.

Raman spectra of CBZ III, PABA I, CBZ/PABA 1:1, CBZ/PABA 2:1, and CBZ/PABA 4:1. 
(a)

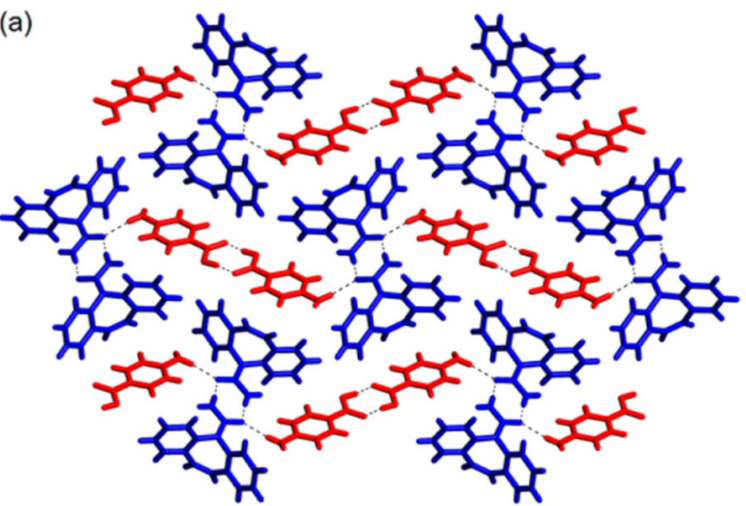

(b)

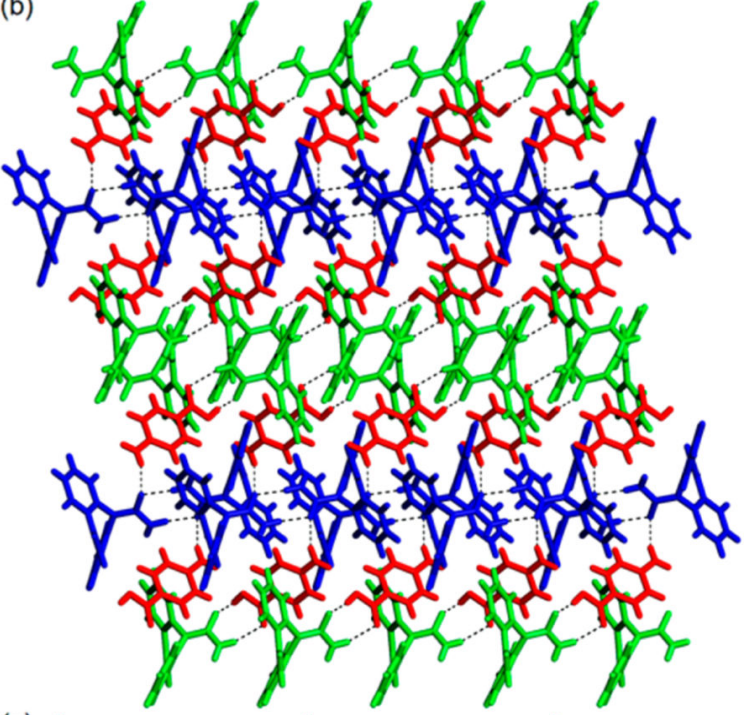

(c)

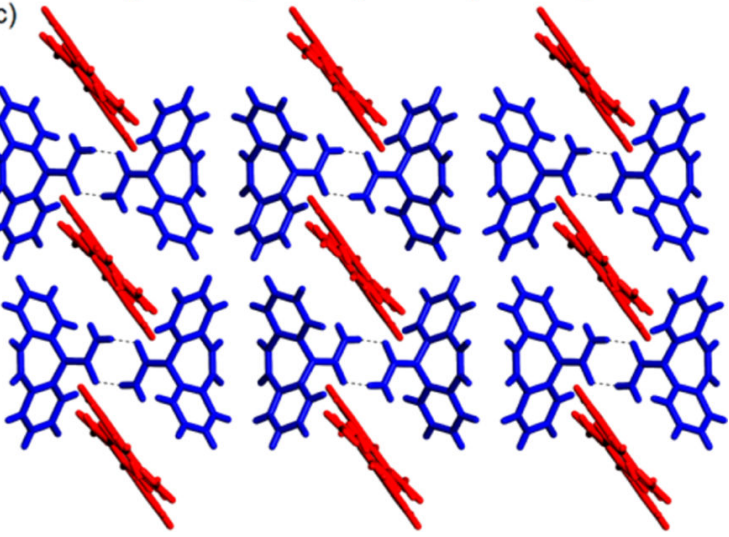

Figure 3.

Packing of (a) CBZ/PABA 1:1, (b) CBZ/PABA 2:1, and (c) CBZ/PABA 4:1 cocrystals. Blue color corresponds to symmetry equivalent homodimerized CBZ. Green color corresponds to symmetry equivalent heterodimerized CBZ. Red color corresponds to PABA. 


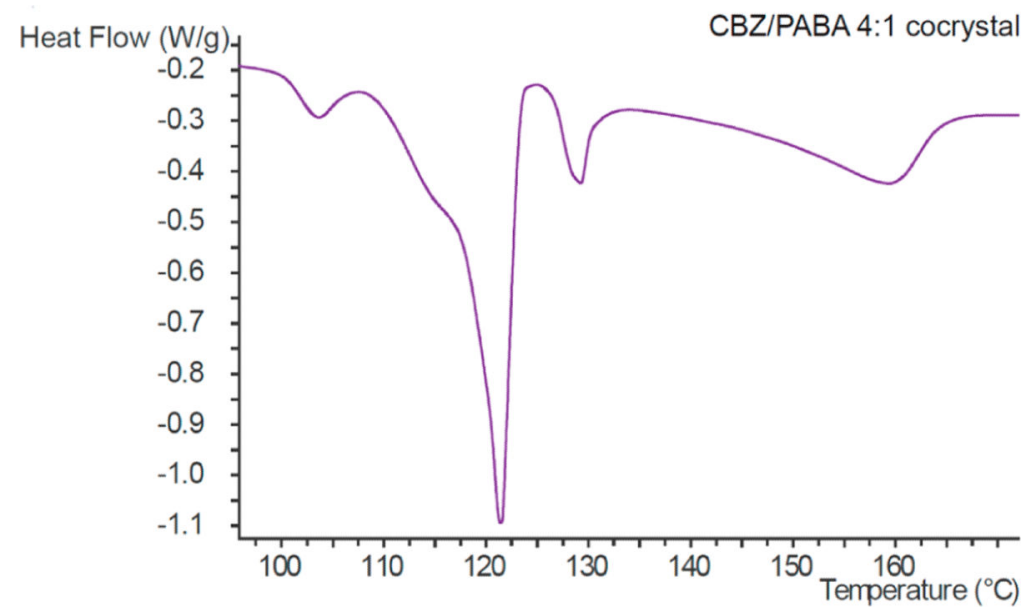

Figure 4.

Differential scanning calorimetry profile of CBZ/PABA 4:1 cocrystal. 
Table 1

Crystal Lattice Comparison of CBZ/PABA Cocrystals ${ }^{a}$

\begin{tabular}{llll}
\hline CBZ/PABA cocrystal stoichiometry & $1: 1$ & $2: 1$ & $4: 1$ \\
lattice syst & monoclinic & monoclinic & monoclinic \\
space group & $P 2_{1} / n$ & $C 2 / c$ & $C 2 / c$ \\
$a(\AA)$ & $5.1909(4)$ & $37.013(3)$ & $20.3857(6)$ \\
$b(\AA)$ & $18.4126(13)$ & $12.1319(9)$ & $5.10210(10)$ \\
$c(\AA)$ & $19.0481(14)$ & $13.5991(10)$ & $26.5935(19)$ \\
$\beta(\operatorname{deg})$ & $97.7740(10)$ & $99.1730(10)$ & $95.583(9)$ \\
$V\left(\AA^{3}\right)$ & 1803.85 & 6028.42 & 2752.87 \\
\hline
\end{tabular}

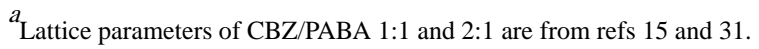




\section{Table 2}

CBZ/PABA Dissolution Rates (Average \pm Standard Error of the Mean) in PBS $^{a}$

\begin{tabular}{lccccc}
\hline & \multicolumn{2}{c}{ CBZ } & & \multicolumn{2}{c}{ PABA } \\
\cline { 2 - 3 } \cline { 5 - 6 } rate of dissoln $\left(\boldsymbol{\mu} \mathbf{m o l} / \mathbf{m i n} / \mathbf{c m}^{2}\right)$ & after $\mathbf{1 ~ h}$ & initial & & after $\mathbf{1 ~ h}$ & initial \\
CBZ III & $7.22 \pm 0.24$ & $21.0 \pm 0.4$ & & \\
CBZ dihydrate & $6.49 \pm 0.10$ & $12.6 \pm 0.7$ & & \\
PABA I & & & & & \\
CBZ/PABA 1:1 & & & & \\
CBZ/PABA 2:1 & $6.45 \pm 0.18$ & $19.4 \pm 1.8$ & & $15.9 \pm 1.2$ & $131 \pm 9$ \\
CBZ/PABA 4:1 & $6.14 \pm 0.15$ & $15.7 \pm 0.8$ & $6.28 \pm 0.29$ & $64.1 \pm 2.3$ \\
\hline
\end{tabular}

${ }^{a}$ All units are in $\mu \mathrm{mol} / \mathrm{min} / \mathrm{cm}^{2}$. Presented data are average values of 10 trials for each form. Pure PABA samples were fully dissolved before $1 \mathrm{~h}$. 
Table 3

CBZ/PABA Dissolution Rates (Average \pm Standard Error of the Mean) in Acetonitrile ${ }^{a}$

\begin{tabular}{lcc}
\hline rate of dissoln $\left(\mathbf{m m o l} / \mathbf{m i n} / \mathbf{c m}^{2}\right)$ & CBZ & PABA \\
CBZ III & $4.62 \pm 0.06$ & \\
CBZ dihydrate & $7.31 \pm 0.08$ & \\
PABA I & & $11.4 \pm 0.1$ \\
CBZ/PABA 1:1 & $5.65 \pm 0.13$ & $5.62 \pm 0.13$ \\
CBZ/PABA 2:1 & $4.31 \pm 0.04$ & $2.17 \pm 0.02$ \\
CBZ/PABA 4:1 & $8.89 \pm 0.13$ & $2.27 \pm 0.04$ \\
\hline
\end{tabular}

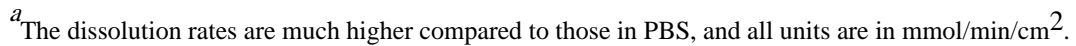

